\title{
Numerical Evaluation of an Isoperimetric Constant
}

\author{
By Z. A. Melzak
}

1. Let $C$ be a rectifiable curve in $E^{n}(n>2)$, let $L(C)$ be its length and $V[H(C)]$ the volume of its convex hull $H(C)$, and consider the problem of maximizing $V[H(C)]$ subject to the condition $L(C)=$ constant. Depending on the parity of $n$ and on whether $C$ is an open arc, or a closed curve, we have four cases to consider: (A) $C$ is closed and $n$ is even, (B) $C$ is open and $n$ is even, (C) $C$ is open and $n$ is odd, (D) $C$ is closed and $n$ is odd.

Under certain restrictive assumptions on $C$ the case (A) (which includes, as a particular example, the isoperimetric problem of the circle) was solved by Schoenberg [1] who proved that then

$$
V[H(C)] / L^{n}(C) \leqq\left[(\pi n)^{n / 2} n !(n / 2) !\right]^{-1}
$$

and the inequality is strict except when $C$ is similar to the hypercircle given parametrically by

$$
x_{2 j-1}(t)=(\sin j t) / j, x_{2 j}(t)=(\cos j t) / j \quad(j=1, \cdots, n / 2)
$$

where $0 \leqq t<2 \pi$. In the case (B) one can use the reflection principle to show that here the maximizing curve is also given by (2) but with $0 \leqq t \leqq \pi$ (a semihypercircle), and the isoperimetric inequality is

$$
V[H(C)] / L^{n}(C) \leqq 2^{n / 2-1}\left[(\pi n / 2)^{n / 2}(n / 2) ! n !\right]^{-1} .
$$

The case (C) with $n=3$ was treated by Egerváry [2]: the isoperimetric inequality is

$$
V[H(C)] / L^{3}(C) \leqq\left(18 \pi \cdot 3^{1 / 2}\right)^{-1}
$$

and it is strict except when $C$ is one turn of a circular helix of pitch $2^{-1 / 2}$. Similar treatment applies in general: the maximizing curve is one turn of a hypercircular helix.

The case (D) with $n=3$ was considered by the author in [3]. Under certain restrictive conditions on $C$ it is possible to express $V[H(C)]$ as an integral:

$$
V[H(C)]=4 \int_{0}^{s / 4} x y z^{\prime} d s
$$

here accent denotes differentiation with respect to the independent variable $s$ which is the arc-length. The integral in (5) is to be maximized subject to the arc-length condition $x^{\prime 2}+y^{\prime 2}+z^{\prime 2}-1=0$, this leads to the Euler-Lagrange equations

$$
4 \lambda^{2} x^{\prime \prime}=x y^{2}, \quad 4 \lambda^{2} y^{\prime \prime}=-y x^{2}, \quad 2 \lambda z^{\prime}=x y,
$$

where $\lambda$ is a constant (the Lagrange multiplier), and the initial conditions are

$$
\text { (7) } \quad x(0)=c_{1}, \quad x^{\prime}(0)=0, \quad y(0)=0, \quad y^{\prime}(0)=c_{2}, \quad z(0)=0 .
$$

Received March 17, 1967. 
Hence, in the isoperimetric inequality for the case (D),

$$
V[H(C)] / L^{3}(C) \leqq B
$$

the equality occurs if and only if $C$ is a periodic solution of (6) and (7). It is shown in [3] that for all $c_{1}$ and $c_{2}$ there is a real-analytic solution $(x, y, z)$ of $(6)$, valid for all $s$, and for each $c$, there is $c_{2}=f\left(c_{1}\right)$, such that the solution is periodic. Unlike in the cases (A), (B), and (C), the maximizing curve in (D) does not appear to be expressible by elementary, or standard transcendental, functions. Our purpose in this note is to compute numerically the corresponding isoperimetric constant $B$ of (8) (which we propose to call the Baggins constant).

2. With suitable scaling the system (6) may be written as

$$
x^{\prime \prime}=-x y^{2}, \quad y^{\prime \prime}=-y x^{2}, \quad z^{\prime}=x y
$$

and the initial conditions are

$$
x(0)=1, \quad x^{\prime}(0)=0, \quad y(0)=0, \quad y^{\prime}(0)=b, \quad z(0)=0 .
$$

The independent variable $t$ is now proportional to the arc-length $s: s=k t$. The constants $k$ and $b$ are very simply related: by (8a) there exists a first integral $x^{\prime 2}+y^{\prime 2}+x^{2} y^{2}=b^{2}$ and since $z^{\prime}=x y$, we have $k=b$. To determine $b$ we consider the differential system $x^{\prime \prime}=-x y^{2}, y^{\prime \prime}=-y x^{2}, x(0)=1, x^{\prime}(0)=y(0)=0$, $y^{\prime}(0)=b$; as shown in [3], the periodic solution of it is symmetric with respect to the lines $x=0, y=0, x \pm y=0$. Writing $x=x(t, b), y=y(t, b)$ and letting $t_{1}$ be the smallest positive value of $t$, for which $x(t, b)=y(t, b)$, we find that $t_{1}$ and $b$ are determined by the equations

$$
x\left(t_{1}, b\right)-y\left(t_{1}, b\right)=0, \quad x^{\prime}\left(t_{1}, b\right)+y^{\prime}\left(t_{1}, b\right)=0 .
$$

For the numerical work we take advantage of the analyticity of $x$ and $y$ in $t$. Let

$$
x(t)=\sum_{0}^{\infty} a_{n} t^{n}, \quad y(t)=\sum_{0}^{\infty} b_{n} t^{n},
$$

then by (8a) and (8b) $x$ turns out to be an even function and $y$ an odd one. Substituting the series (10) into $(8 \mathrm{a})$ we get

$$
\begin{aligned}
& a_{n+2}=-[(n+1)(n+2)]^{-1} \sum_{i+j+k=n} a_{i} b_{j} b_{k}, \\
& b_{n+2}=-[(n+1)(n+2)]^{-1} \sum_{i+j+k=n} b_{i} a_{j} a_{k} .
\end{aligned}
$$

Suppose next that for $m=0,1, \cdots, n$

$$
\left|a_{m}\right| \leqq \alpha \lambda^{m}, \quad\left|b_{m}\right| \leqq \alpha \lambda^{m} .
$$

Then, by (11) and by the parities of $x$ and $y$, we have the estimates

$$
\left|a_{n+2}\right| \leqq[(n+1)(n+2)]^{-1} \alpha^{3} \lambda^{n} N(n), \quad\left|b_{n+2}\right| \leqq[(n+1)(n+2)]^{-1} \alpha^{3} \lambda^{n} M(n)
$$

where $N(n)$ (resp. $M(n)$ ) is the number of representations of an even (resp. odd) integer $n$ in the form $i+j+k$, with $0 \leqq i, j, k$, and $i$ is even (resp. odd) while $j$ and $k$ are odd (resp. even). Therefore, for $n \geqq 2$

$$
\left|a_{n+2}\right| \leqq \alpha^{3} \lambda^{n} / 4, \quad\left|b_{n+2}\right| \leqq \alpha^{3} \lambda^{n} / 4 .
$$


Hence (12) will have been proved inductively to hold for all $m$ if $1 \leqq \alpha, b \leqq a \lambda$, (initialization, by (8b) and (12)), $\alpha \leqq 2 \lambda$, (induction, by (12) and (13)).

To optimize the estimates (12) we make $\lambda$ possibly small by letting $\alpha=2 \lambda$ and $b=\alpha \lambda$, to get $\lambda=(b / 2)^{1 / 2}, \alpha=(2 b)^{1 / 2}$ so that (12) becomes

$$
\left|a_{m}\right| \leqq(2 b)^{1 / 2}(b / 2)^{m / 2}, \quad\left|b_{m}\right| \leqq(2 b)^{1 / 2}(b / 2)^{m / 2} .
$$

Now we determine the constants $b$ and $t_{1}$ from (9), by taking a square grid of values for $b$ and $t_{1}$ (11 by 11), solving the differential system numerically, and refining then the grid over the square where (9) appears to have its roots. After this is repeated two or three times, we may evaluate $x$ and $y$ numerically for a grid of three points only and fit planes through these triples of points. In this way $b$ and $t_{1}$ are determined to be

$$
b=0.92114882, \quad t_{1}=1.22036757 .
$$

Observe that by (14) the power-series (10) converge for $t<(2 / b)^{1 / 2}=1.473 \cdots$ which exceeds the calculated value $t_{1}$ so that the solution procedure by using power series is justified on the interval $0 \leqq t \leqq t_{1}$. Moreover, the estimates (14) lead to simple error estimates for truncating the power series for $x$ and $y$ and their derivatives.

3. The evaluation of $B$ is now simple. First, we notice the four-fold inversion symmetry of the curve $C$ given by $(8 \mathrm{a})$ and $(8 \mathrm{~b})$ : if it is rotated by $\pm 90^{\circ}$ about the $z$-axis the effect is the same as reflecting $C$ in the plane $z=z_{\max } / 2$. Therefore, the arc of $C$ given by $0 \leqq t \leqq t_{1}$ (where $t_{1}$ is given by (15)) has the length $b t_{1}=L(C) / 8$, and by (5) the volume of $H(C)$ is

$$
V[H(C)]=8 \int_{0}^{b t_{1}} x y z^{\prime} d t .
$$

The integral above is easily computed by integrating the product of truncated power series for $x, y$, and $z^{\prime}$, and we get finally $B=0.0031816877$.

4. The author is obliged to Professor H. B. Keller, of the Courant Institute of Mathematical Sciences, for several suggestions concerning numerical procedures. Thanks are also expressed to the National Research Council of Canada for its support in the form of a Senior Fellowship, and to the A.E.C. Computing and Applied Mathematics Center at the Courant Institute of Mathematical Sciences, New York University, for the use of their CDC 6600 computing machine during the academic year the author spent at the Institute. Finally, the author is grateful to the referee for pointing out that the Eqs. (8a) were obtained (though apparently unpublished) by G. Pólya in 1951 or 1952.

\section{University of British Columbia} Vancouver, B.C. Canada

1. I. J. Schoenberg, "An isoperimetric inequality for closed curves convex in even-dimensional Euclidean spaces," Acta Math., v. 91, 1954, pp. 143-164. MR 16, 508.

2. E. Egerváry, "On the smallest convex cover of a simple arc of space-curve," Publ. Math. Debrecen, v. 1, 1949, pp. 65-70. MR 12, 46 .

3. Z. A. MELzaK, "The isoperimetric problem of the convex hull of a closed space curve," Proc. Amer. Math. Soc., v. 11, 1960, pp. 265-274. MR $22 \# 7058$. 